\title{
Validación pronóstica según los criterios de la GesEPOC 2017
}

\section{Carlos Cabrera López ${ }^{\mathrm{a}, *}$, Ciro Casanova Macario ${ }^{\mathrm{b}}$, José María Marín Trigo ${ }^{\mathrm{c}}$, Juan P. de-Torres ${ }^{\mathrm{d}}$, Rebeca Sicilia Torres $^{a}$, Jesús María González ${ }^{\mathrm{e}}$, Francesca Polverino ${ }^{\mathrm{f}}$, Miguel Divo ${ }^{\mathrm{f}}$, Víctor Pinto Plata ${ }^{\mathrm{g}}$, Javier Zulueta $^{\mathrm{d}}$, Francisco Javier Callejas ${ }^{\mathrm{h}}$ y Bartolomé Celli ${ }^{\mathrm{f}}$}

a Servicio de Neumología, Hospital Universitario de Gran Canaria Dr. Negrín, Las Palmas de Gran Canaria, España

b Servicio de Neumología, Hospital Universitario Nuestra Señora de la Candelaria, Santa Cruz de Tenerife, España

' Servicio de Neumología, Hospital Universitario Miguel Servet, Zaragoza, España

d Servicio de Neumología, Clínica Universitaria de Navarra, Pamplona, España

e Unidad de Investigación, Hospital Universitario de Gran Canaria Dr. Negrín, Las Palmas de Gran Canaria, España

f Pulmonary and Critical Care Service, Brigham and Women's Hospital, Boston, Massachusetts, Estados Unidos

g Pulmonary and Critical Care Division, Baystate Medical Center, Springfield, Massachusetts, Estados Unidos

h Complejo Hospitalario Universitario de Albacete, Albacete, España

\section{INFORMACIÓN DEL ARTÍCULO}

\section{Historia del artículo:}

Recibido el 7 de julio de 2018

Aceptado el 3 de diciembre de 2018

on-line el $\mathrm{xxx}$

\section{Palabras clave:}

EPOC

GesEPOC

Pronóstico

Riesgo

Mortalidad

\section{R E S U M E N}

Introducción: La Guía española de la EPOC (GesEPOC) ha sido recientemente modificada. El objetivo de este trabajo es valorar la clasificación y el pronóstico de los enfermos según la nueva clasificación de la gravedad.

Métodos: Se siguió a 700 enfermos con EPOC (83,9\% varones) durante un periodo medio de 5 años en hospitales españoles y de EE. UU. Se midieron datos antropométricos, función pulmonar, disnea medida con la escala mMRC, así como exacerbaciones y los índices de BODE y Charlson. Se clasificaron según el riesgo proporcionado por GesEPOC y se valoró el pronóstico a 5 años.

Resultados: Los pacientes tenían una edad media de $66 \pm 9,6$ años y un $\mathrm{FEV}_{1} \%$ de $59,7 \pm 20,2$. El 40,43\% de la muestra se encontraba en bajo riesgo. Los sujetos del grupo de alto riesgo presentaban un índice de BODE significativamente mayor que los de bajo riesgo $(2,92 \pm 0,66$ vs. 0,52 $\pm 1,91, \mathrm{p}<0,001)$. El índice de Charlson fue similar entre ambos grupos. La mortalidad a 60 meses en el grupo de alto riesgo fue significativamente mayor que en el de bajo riesgo ( $31,7 \%$ vs. $15.5 \%$, p < 0,001). Tanto la disnea como el $\mathrm{FEV}_{1} \%$ fueron también predictores independientes de mortalidad ( $\mathrm{p}<0,001$ ), siendo cada uno de ellos no inferior prediciendo mortalidad que el conjunto de los criterios del grupo de alto riesgo de GesEPOC. Conclusiones: La nueva clasificación de la gravedad de GesEPOC predice la mortalidad de forma adecuada. No obstante, tanto el $\mathrm{FEV}_{1} \%$ como la disnea tienen la misma potencia para predecir mortalidad.

(C) 2018 SEPAR. Publicado por Elsevier España, S.L.U. Todos los derechos reservados.

\section{Prognostic Validation Using GesEPOC 2017 Severity Criteria}

\section{A B S T R A C T}

Introduction: The Spanish COPD guidelines (GesEPOC) have been recently modified. The aim of this study is to assess this revision and evaluate the prognosis of patients according to the new classification of severity.

Methods: A total of 700 COPD patients ( $83.9 \%$ men) were prospectively followed up for a mean period of 5 years in tertiary hospitals in Spain and the USA. Anthropometric data, lung function tests, dyspnea (according to the mMRC scale), BODE and Charlson index were collected. We calculated mortality at 5 years following the risk criteria proposed by the new GesEPOC.

Results: Mean age was $66 \pm 9.6$ years and mean $\mathrm{FEV}_{1} \%$ was $59.7 \pm 20.2$. The proportion of patients in the low-risk group was $40.43 \%$. Patients in the high-risk group had a significantly higher BODE index than those in the low-risk group $(2.92 \pm 0,66$ vs. $0.52 \pm 1.91, \mathrm{p}<0.001)$, while the Charlson index score was

\footnotetext{
* Autor para correspondencia.

Correo electrónico: ccablopn@gmail.com (C. Cabrera López).
} 
similar in both groups. Mortality at 60 months was significantly higher in the high-risk group (31.7\% vs. $15.5 \%, \mathrm{p}<0.001)$. Dyspnea and $\mathrm{FEV}_{1} \%$ were also independent predictors of mortality $(\mathrm{p}<0.001)$, and neither was inferior to the risk classification proposed by GesEPOC.

Conclusions: The new severity index proposed by GesEPOC accurately predicts 5-year mortality. However, dyspnea and $\mathrm{FEV}_{1} \%$ have the same strength in predicting mortality.

(c) 2018 SEPAR. Published by Elsevier España, S.L.U. All rights reserved.

\section{Introducción}

La enfermedad pulmonar obstructiva crónica (EPOC) es una de las principales causas de morbimortalidad a nivel mundial ${ }^{1}$, siendo además una enfermedad con alta prevalencia e infradiagnóstico en nuestro país ${ }^{2}$. La primera Guía española de la EPOC $(\text { GesEPOC })^{3}$ se realizó en 2012 en el marco del Plan de calidad del Sistema Nacional de Salud y la estrategia en EPOC del Ministerio de Sanidad, Igualdad y Política Social. En esta versión inicial tanto la clasificación del paciente como su tratamiento venían definidos según los fenotipos existentes en la EPOC. Esta primera versión de la guía ha sido modificada recientemente con un cambio sustancial en el acercamiento al enfermo. De una clasificación inicial por fenotipos se ha pasado a una basada en el riesgo, existiendo un grupo de bajo riesgo y otro de alto riesgo ${ }^{4}$. El riesgo viene definido por la función pulmonar, la disnea y las exacerbaciones, tal y como hacía previamente el documento de la Global Initiative for Obstructive Lung Disease (GOLD) ${ }^{5}$. No obstante, los criterios para formar parte de los diferentes grupos de riesgo son distintos a los propuestos por la GOLD. Se ha decidido suprimir los índices multidimensionales de gravedad establecidos en la anterior versión (BODEx $)^{6}$ para realizar una propuesta de clasificación más clínica y sencilla. Según el grupo de riesgo donde se encuentre el enfermo, se propone dar uno o dos broncodilatadores. Posteriormente se propone un acercamiento farmacológico según los fenotipos (con la excepción del fenotipo mixto, que llevaría corticoides inhalados de entrada). Esta concepción de la guía clínica probablemente permita una mayor utilización de la misma en atención primaria. No obstante, esta nueva visión en la clasificación del riesgo no ha sido aún evaluada, y poco sabemos de cómo afecta a la distribución de los enfermos y a su pronóstico a largo plazo.

Este estudio de pacientes con EPOC evaluados prospectivamente valora la distribución de los mismos según el riesgo y sus componentes individuales. Además, valora el pronóstico a largo plazo de los pacientes según la nueva clasificación GesEPOC.

\section{Métodos}

Los participantes de este estudio son parte de la cohorte BODE. Esta cohorte consta de enfermos EPOC seguidos en el ámbito de consultas de neumología de forma consecutiva entre 1997 y 2017. Los criterios de inclusión y exclusión han sido previamente descritos $^{7}$. En resumen, es una cohorte longitudinal, prospectiva y multicéntrica con al menos un control anual. En todos los pacientes se miden las características antropométricas y se realiza un examen físico. Se les realiza una espirometría siguiendo los criterios de la Sociedad Americana del Tórax y la Sociedad Europea de Respiratorio y se realiza el diagnóstico de EPOC siguiendo los criterios establecidos internacionalmente ${ }^{8,9}$. Todos los pacientes de la cohorte tienen al menos un índice de paquetes/año (IPA) mayor de $10 \mathrm{y}$ han estado estables un periodo mínimo de 6 semanas previo a las pruebas. Las exacerbaciones se definen como cualquier episodio de empeoramiento de la disnea, tos o expectoración que requiera antibióticos o corticoides sistémicos ${ }^{10}$. Las exacerbaciones eran reportadas por el paciente y confirmadas posteriormente con la historia clínica electrónica. Las exacerbaciones se clasificaron en moderadas si eran ambulatorias y tratadas con corticoides orales o con antibióticos, o graves si requerían hospitalización. La disnea se midió con la escala modificada del Medical Research Council (mMRC $)^{11}$. Se calculó a su vez el índice de BODE, así como el índice de Charlson ${ }^{7,12}$. Las comorbilidades fueron recogidas usando las enfermedades referidas por el enfermo y revisando la historia clínica electrónica. Se seleccionó el test de la marcha de los 6 minutos (6 MWT) como el mejor de dos recorridos separados al menos $30 \mathrm{~min}^{13}$. Se recogió la mortalidad global hasta febrero de 2017. Se analizó a su vez mortalidad específica por EPOC, causa respiratoria no asociada a EPOC, cáncer de pulmón, causas cardiovasculares, otras causas y causas desconocidas. Todos los participantes recibieron un tratamiento óptimo según las guías internacionales del momento y firmaron los consentimientos informados previamente aprobados por los comités de ética de cada centro participante.

\section{GesEPOC 2017}

Se clasificó a los enfermos en alto y bajo riesgo siguiendo los criterios actuales de la guía GesEPOC (cualquiera de los siguientes criterios haría que el enfermo entrara en el grupo de alto riesgo: $\mathrm{FEV}_{1} \%<50 \%$, disnea $\geq 2$ si está con tratamiento, 2 o más exacerbaciones ambulatorias o al menos una hospitalaria el año anterior). Posteriormente se calculó la proporción de sujetos que eran clasificados en alto riesgo según los diferentes criterios individuales que lo componen, así como aquellos sujetos que pertenecen al alto riesgo por suma de criterios. Se calculó la comorbilidad por grupos y la mortalidad a 5 años según las diferentes causas.

\section{Estadística}

Se ha utilizado el test de Shapiro-Wilk para comprobar la normalidad de los datos de las variables cuantitativas. Se calculó la media y desviación típica para aquellas variables que siguen una distribución normal. Aquellas variables que no presentan una distribución normal se describen mediante la mediana y el rango de los cuartiles 1 y $3\left(Q_{1}-Q_{3}\right)$. Para comparar variables cualitativas se ha utilizado el test exacto de Fisher. Se han realizado modelos de regresión logística para predecir la mortalidad a los 60 meses del modelo de alto riesgo de GesEPOC y de las 4 variables que lo conforman, diferenciándose exacerbaciones ambulatorias y hospitalarias. Todos los modelos han sido ajustados por edad y género. Para medir la capacidad de predicción de estos modelos se han calculado las curvas ROC y sus respectivas áreas bajo la curva (AUC). Las variables cuantitativas se compararon mediante el test de la t de Student. Se utilizó a su vez el método de Kaplan-Meier para representar gráficamente las curvas de supervivencia y el test de log-rank para comparar si hay diferencias entre dichas curvas. Valores de $\mathrm{p}<0,05$ se han considerado significativos. El programa estadístico utilizado ha sido R Core Team $(2018)^{14}$.

\section{Resultados}

Las características de la muestra se exponen en la tabla 1. Los 700 pacientes tenían una edad media de $66 \pm 9,6$ años y un $\mathrm{FEV}_{1} \%$ 
Tabla 1

Características de la muestra de enfermos con EPOC

\begin{tabular}{|c|c|}
\hline $\mathrm{N}$ & 700 \\
\hline Varones, $n(\%)$ & $587(83,98)$ \\
\hline Edad $($ media $\pm D E)$ & $66,4 \pm 9,26$ \\
\hline$I M C($ media $\pm D E)$ & $27,43 \pm 4,9$ \\
\hline Fumadores activos, $n(\%)$ & $236(33,76)$ \\
\hline$I P A($ media $\pm D E)$ & $60,66 \pm 31,16$ \\
\hline$F E V_{1} \%($ media $\pm D E)$ & $59,71 \pm 20,21$ \\
\hline GOLD1, $n(\%)$ & $122(17,43)$ \\
\hline GOLD2, $n(\%)$ & $348(49,71)$ \\
\hline GOLD3, $n(\%)$ & $179(25,57)$ \\
\hline GOLD4, $n(\%)$ & $51(7,29)$ \\
\hline Índice de Charlson (media $\pm D E$ ) & $2,25 \pm 2,31$ \\
\hline Índice de BODE (media $\pm D E)$ & $1,93 \pm 1,93$ \\
\hline \multicolumn{2}{|l|}{ Clasificación según GOLD, $n$ (\%) } \\
\hline$A$ & $373(53,29)$ \\
\hline B & $184(26,29)$ \\
\hline $\mathrm{C}$ & $60(8,57)$ \\
\hline $\mathrm{D}$ & $83(11,86)$ \\
\hline Disnea $(\text { media } \pm D E)^{\mathrm{a}}$ & $1,34 \pm 1,09$ \\
\hline Disnea $<2, n(\%)$ & $524(74,86)$ \\
\hline Exacerbadores, $n(\%)^{\mathrm{b}}$ & $174(25,63)$ \\
\hline Exacerbador hospitalario, $n(\%)^{c}$ & $66(9,43)$ \\
\hline Exacerbador ambulatorio, $n(\%)^{\mathrm{d}}$ & $108(15,43)$ \\
\hline Alto riesgo según GesEPOC, $n(\%)$ & $417(59,57)$ \\
\hline Exitus, $n(\%)$ & $176(25,14)$ \\
\hline Exitus pulmonar, $n(\%)$ & $88(12,57)$ \\
\hline Exitus EPOC, $n(\%)$ & $51(7,29)$ \\
\hline Exitus cardiovascular, $n(\%)$ & $22(3,14)$ \\
\hline Exitus cáncer pulmón, $n$ (\%) & $33(4,71)$ \\
\hline Tiempo de seguimiento (media $\pm D E$ ) (meses) & $53,61 \pm 34,44$ \\
\hline
\end{tabular}

BODE: índice de masa corporal, obstrucción al flujo aéreo, disnea y capacidad de ejercicio; DE: desviación estándar; GesEPOC: Guía española de la EPOC; GOLD: Global Initiative for Chronic Obstructive Disease; IMC: índice de masa corporal; IPA: índice de paquetes/año.

a Disnea medida por la modified Medical Research Council (mMRC).

b Sujetos con 2 o más exacerbaciones que hayan requerido antibióticos o corticoides sistémicos o al menos una exacerbación hospitalaria en el año anterior.

c Sujetos que solo han presentado una o más exacerbaciones hospitalarias, pero no exacerbaciones ambulatorias.

d Sujetos que han presentado 2 o más exacerbaciones ambulatorias pero que no han tenido exacerbaciones hospitalarias.

de $59,7 \pm 20,2$. La mayoría de los enfermos presentaban un grado de obstrucción moderado o grave. Más del $80 \%$ eran varones y un tercio de la muestra era fumadora activa. El índice de paquetes/año era alto, con una media de $60 \pm 31,16$.

\section{Clasificación según GesEPOC}

El 40,43\% de la muestra se encontraba en el grupo de bajo riesgo. En el alto riesgo, un $38,14 \%$ presentaba una disnea mayor o igual a 2 en la escala mMRC y un 15,43\% exacerbaciones ambulatorias, mientras que solamente un $9,43 \%$ presentaba exacerbaciones hospitalarias (total de sujetos exacerbadores: 174 , un $24,86 \%$ de la muestra). Los sujetos con un $\mathrm{FEV}_{1}$ por debajo del $50 \%$ eran un $32,86 \%$. Los enfermos clasificados de alto riesgo que solo presentaban un factor de riesgo eran el 30,71\%, los que tenían 2 factores de riesgo eran un $19,86 \%$, mientras que los que tenían 3 o 4 factores de riesgo (se separaron exacerbaciones ambulatorias de hospitalarias) eran un 8,14 y un $0,86 \%$, respectivamente.

\section{Comorbilidades y gravedad}

Los sujetos englobados en el grupo de alto riesgo presentaban un índice de BODE significativamente mayor que los de bajo riesgo $(2,92 \pm 0,66$ vs. $0,52 \pm 1,91$, respectivamente, $\mathrm{p}<0,001)$. El índice de BODE era mayor en todos los sujetos que presentaban algún factor de riesgo, independiente del mismo. El índice de Charlson no difería entre los grupos de alto y bajo riesgo $(2,21 \pm 2,38$ vs. $2,27 \pm 2,26$,

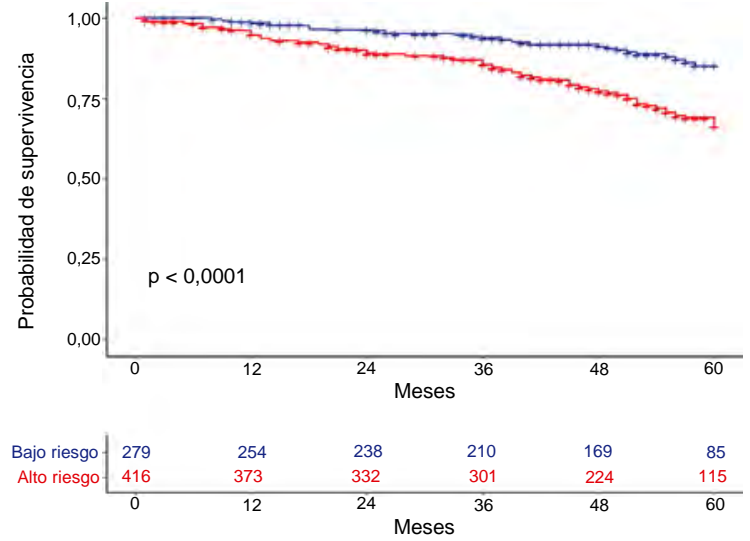

Figura 1. Kaplan-Meier de mortalidad a 60 meses según el grupo de riesgo de la guía GesEPOC.

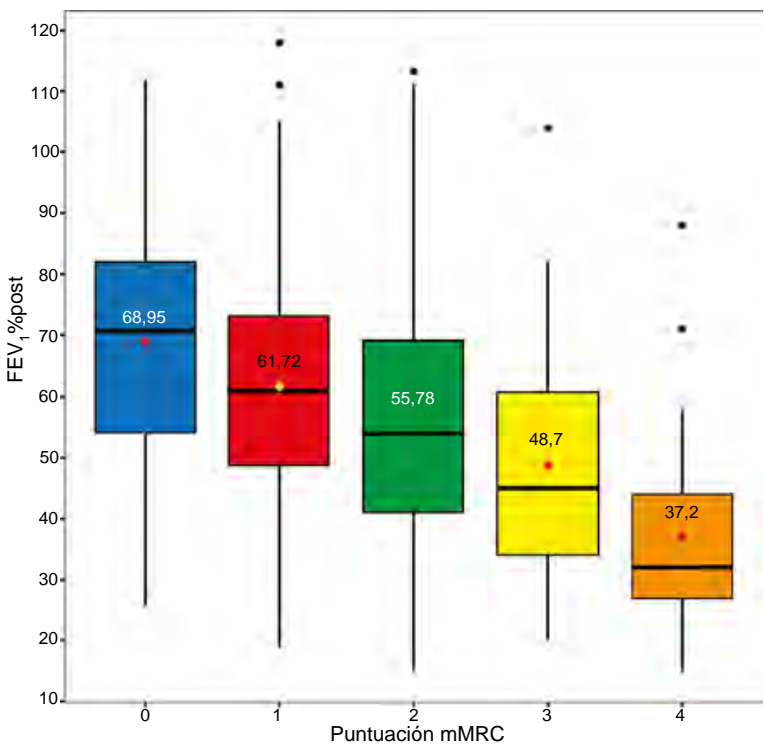

Figura 2. Relación lineal entre el $\mathrm{FEV}_{1} \%$ y la disnea medida con la escala modified Medical Research Council (mMRC).

respectivamente, $\mathrm{p}=0,57)$. Sí existe una relación marginalmente significativa entre los sujetos que exacerbaban de forma ambulatoria con un aumento del índice de comorbilidades $(\mathrm{p}=0,07)$.

\section{Mortalidad}

La mortalidad total a los 60 meses fue de un 25,4\%, siendo significativamente mayor en el grupo de alto riesgo que en el de bajo riesgo (31,7\% vs. 15,5\%, respectivamente, $\mathrm{p}<0,001)$ (fig. 1 ). Tanto la disnea, las exacerbaciones hospitalarias y el $\mathrm{FEV}_{1} \%$ estuvieron relacionados significativamente con mortalidad $(\mathrm{p}<0,001$, $\mathrm{p}=0,0012$ y $\mathrm{p}<0,001$, respectivamente). La disnea y el $\mathrm{FEV}_{1} \%$ presentaron una relación inversamente proporcional con un índice de Spearman de $-0,39$ (fig. 2). Las exacerbaciones ambulatorias no estuvieron relacionadas con la mortalidad $(p=0,24)$. La mortalidad por causa cardiovascular no estaba aumentada en los sujetos de riesgo alto con relación a los de riesgo bajo (3,8\% vs. $2,1 \%$, respectivamente, $\mathrm{p}=0,27)$. Sí estaba aumentada la mortalidad relacionada con causa pulmonar (6,7\% en el riesgo bajo vs. $16,5 \%$ en el riesgo alto, $\mathrm{p}<0,001)$ o con EPOC ( $1,8 \%$ en el riesgo bajo vs. $11 \%$ en el riesgo alto, $\mathrm{p}<0,001)$. La mortalidad cardiovascular estaba incrementada en los sujetos que presentan un $\mathrm{FEV}_{1}$ menor del $50 \%$ como factor de riesgo (2,1\% vs. $5,2 \%$ para bajo y alto riesgo, 
Tabla 2

Número de factores de riesgo según la clasificación del riesgo por GesEPOC $\left(\mathrm{FEV}_{1} \%\right.$, disnea medida con la escala mMRC, exacerbaciones hospitalarias y exacerbaciones ambulatorias) y su relación con mortalidad

\begin{tabular}{llll}
\hline & \multicolumn{2}{c}{ Exitus } & Total \\
\cline { 2 - 3 } Número de factores de riesgo & No & Sí & \\
\hline Riesgo bajo, n (\%) & $239(84,5)$ & $44(15,5)$ & $283(40,4)$ \\
1 factor de riesgo, n (\%) & $160(74,4)$ & $55(25,6)$ & $215(30,7)$ \\
2 factores de riesgo, n(\%) & $92(66,2)$ & $47(33,8)$ & $139(19,9)$ \\
3 factores de riesgo, n (\%) & $32(56,1)$ & $25(43,9)$ & $57(8,1)$ \\
4 factores de riesgo, n (\%) & $1(16,7)$ & $5(83,3)$ & $6(0,9)$ \\
Total & 254 & 176 & 700 \\
\hline
\end{tabular}

GesEPOC: Guía española de la EPOC; mMRC: modified Medical Research Council.

respectivamente, $p=0,048$ ). La mortalidad por cáncer de pulmón fue independiente de la clasificación del riesgo. La mortalidad aumenta de forma lineal según el número de factores de riesgo que tiene cada enfermo $(\mathrm{p}<0,001)$. Esta relación está expresada en la tabla 2. La predicción de mortalidad según la estratificación del riesgo por GesEPOC, medida por las probabilidades obtenidas mediante el modelo de regresión logística, no fue significativamente mejor que la realizada exclusivamente por la disnea, por el $\mathrm{FEV}_{1} \%$ o por las exacerbaciones hospitalarias (fig. $3 \mathrm{~A} \mathrm{y} \mathrm{B}$ ).

\section{Discusión}

En este estudio de pacientes con EPOC seguidos prospectivamente y clasificados siguiendo los criterios recomendados por las guías GesEPOC de 2017 se observa que el 40,43\% de los pacientes se sitúan en el grupo de bajo riesgo, mientras que el 59,57\% quedarían clasificados en el grupo de alto riesgo. El grupo de alto riesgo tiene una mayor mortalidad, a pesar de no tener diferencias en las comorbilidades, que el grupo de bajo riesgo. Los elementos que definen el grupo de alto riesgo ( $\mathrm{FEV}_{1}$, disnea y exacerbaciones) presentan relación con la mortalidad de forma independiente, a excepción de las exacerbaciones ambulatorias. Datos de otros estudios avalan parte de nuestros resultados, tales como la mortalidad y, en menor medida, la distribución de los pacientes ${ }^{15}$.

El factor que más se asocia a mortalidad es la función pulmonar, posteriormente la disnea (sin que existan diferencias estadísticas entre estas dos variables) y las exacerbaciones hospitalarias, que presentan una capacidad de predecir mortalidad similar al conjunto de los diferentes criterios que conforman el grupo de alto riesgo. La adición de factores de riesgo aumenta el mal pronóstico de los enfermos, siendo diferente en los que presentan exclusivamente una variable de los que presentan 2, 3 o las 4 variables. Este diferente pronóstico según el número de variables diferencia aún más a los pacientes que están dentro del grupo de alto riesgo, subdividiéndolo en gravedad.
Ha sido previamente descrito el aumento de la prevalencia de las enfermedades cardiovasculares en los sujetos que presentan disnea, no obstante, en este trabajo, la mortalidad asociada al grupo de alto riesgo no se debe a enfermedades cardiovasculares, sino a causa pulmonar y por EPOC. La única asociación con comorbilidad vascular la da el $\mathrm{FEV}_{1} \%$, dato que ha sido previamente publicado ${ }^{16-18}$. Es por tanto poco probable que la potencia pronóstica en mortalidad de la disnea se deba a su asociación con enfermedades cardiovasculares.

El nuevo sistema de GesEPOC para la clasificación de la gravedad se aleja de lo propuesto en la anterior guía. El acercamiento actual, más sencillo, parece tener una buena concordancia con el pronóstico. La inclusión de la función pulmonar es un elemento de coherencia en la clasificación de los enfermos con EPOC para atención especializada. Sin embargo, es llamativo que no existe superioridad en la predicción de mortalidad entre lo propuesto por la guía o simplemente la disnea o el $\mathrm{FEV}_{1} \%$ como elementos independientes. Esto se debe probablemente a la relación lineal entre estos dos parámetros, principales componentes de esta clasificación. Esta relación lineal no es perfecta, pero tampoco se debe menospreciar.

La potencia del $\mathrm{FEV}_{1} \%$ para predecir la mortalidad es mayor en este trabajo que la que proporciona la disnea. No obstante, la relación entre la mortalidad cardiovascular y el $\mathrm{FEV}_{1} \%$ puede estar influyendo en esta diferencia. La disnea sigue siendo un predictor de mortalidad para causa pulmonar y para EPOC potente y fiable. Podríamos preguntarnos si en ámbitos como el de atención primaria, con dificultades en la realización de la espirometría, sería posible utilizar la disnea como marcador de gravedad y guía principal del tratamiento de la enfermedad una vez que ha sido diagnosticada ${ }^{19}$.

Es interesante observar como las exacerbaciones, cuando se analizan de forma conjunta ambulatorias y hospitalarias, no presentan relación con la mortalidad. La denominación de exacerbador frecuente es probablemente la causa de este fenómeno. Es bien conocida la relación de la mortalidad con las exacerbaciones hospitalarias ${ }^{20}$ pero no la relación entre mortalidad y las exacerbaciones ambulatorias o moderadas, que hasta ahora no ha podido ser demostrada. Para añadir más fortaleza a la clasificación se deberían obviar las exacerbaciones ambulatorias, quedando exclusivamente las hospitalarias. Esto no quiere decir que no haya que tratar o prevenir las exacerbaciones ambulatorias, que son causa de disminución de la calidad de vida y del uso de los recursos sanitarios ${ }^{21,22}$. Un dato a destacar es que la cantidad de enfermos que están en el grupo de alto riesgo exclusivamente por exacerbaciones ambulatorias es solamente del $4,29 \%$, lo que minimiza el impacto en el sistema de clasificación. El resto de los sujetos exacerbadores, o bien exacerban de forma hospitalaria (grave), o bien presentan exacerbaciones ambulatorias y otro factor de riesgo añadido.
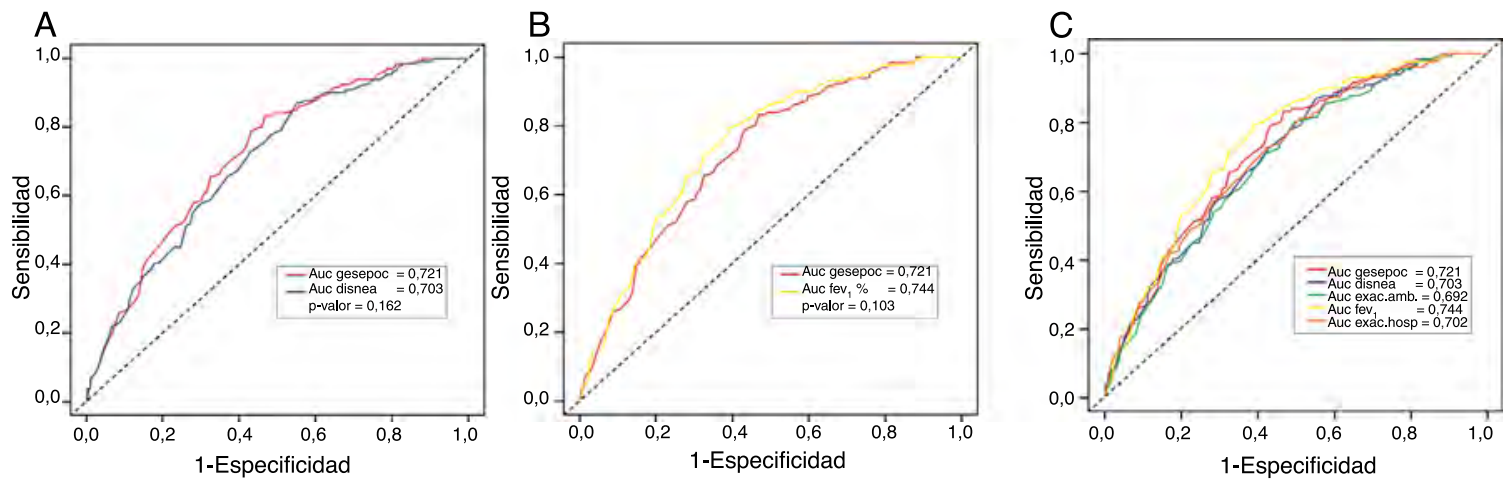

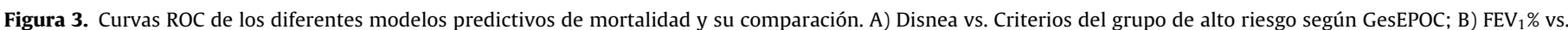
Criterios del grupo de alto riesgo según GesEPOC; C) Criterios del grupo de alto riesgo según GesEPOC vs. Criterios del grupo de alto riesgo por separado. 
En nuestra muestra, comparando la clasificación de la GesEPOC con la clasificación realizada según el documento GOLD, encontramos una diferencia de casi un $40 \%$ en la prevalencia entre los grupos de alto riesgo de la GOLD (C y D) y el grupo de alto riesgo de GesEPOC. Si realizamos esta comparación con los grupos B y D (los que más mortalidad conllevan en la clasificación GOLD) ${ }^{23}$, la diferencia se reduce -aunque sigue siendo alta- al 21\%. Esta diferencia viene dada probablemente por la presencia de un grupo de sujetos que estarían clasificados en el grupo A de la GOLD pero que tendrían un $\mathrm{FEV}_{1}$ menor del $50 \%$. Estas discordancias influyen claramente en el tratamiento propuesto por ambas guías para el manejo de los enfermos; un mínimo de un $20 \%$ más de pacientes tendrían doble broncodilatación si se usa la GesEPOC, pudiendo ser mucho mayor este porcentaje (hasta más de un 50\% de la muestra) si los enfermos que se clasifican en los grados B y C de la GOLD estuvieran tratados con las recomendaciones de primera elección (un broncodilatador de larga acción en el grupo B y un LAMA en el C). Desde nuestro punto de vista, el acercamiento de GesEPOC está más ligado a la realidad y a la práctica clínica habitual desarrollada en nuestro medio. La exclusión del $\mathrm{FEV}_{1} \%$ en la cuadrícula de GOLD ha desvirtuado su utilidad clínica y reducido su potencia para predecir el pronóstico de la enfermedad.

Nuestro estudio presenta algunas limitaciones. En primer lugar, la muestra tiene pocas mujeres. En nuestro trabajo no llegan al $20 \%$ de los participantes, sin embargo, el número de mujeres seguidas en este estudio, que fue de 113, no es despreciable y además sus resultados son similares a los encontrados en los hombres. En segundo lugar, las comorbilidades cardiovasculares no están desglosadas ni especificadas. A pesar de usar un índice de comorbilidad ampliamente validado, en este estudio no se diferencia entre comorbilidades cardiovasculares (ni sus diferentes tipos), oncológicas u otras. No sabemos la cantidad de comorbilidad cardiovascular que se encuentra presente entre los sujetos con mucha disnea. Se ha demostrado previamente que la comorbilidad cardiovascular puede llegar hasta un $20 \%$ en los sujetos con mucha disnea ${ }^{24}$, sin embargo, no parece que esto esté produciendo ningún sesgo en el trabajo ya que no existe asociación entre mortalidad cardiovascular y disnea en este estudio.

En resumen, este estudio demuestra que la clasificación actual de la gravedad de la guía GesEPOC tiene capacidad discriminativa en el pronóstico de los pacientes con EPOC. Este grupo presenta una mortalidad muy superior al de bajo riesgo y, dentro de este grupo de alto riesgo, la mortalidad aumenta a medida que se suman diferentes factores de riesgo. No obstante, las exacerbaciones, tal y como están definidas en la actualidad, no están relacionadas con la mortalidad. Tanto la disnea como el $\mathrm{FEV}_{1} \%$ de forma individual tienen la misma potencia pronóstica que el índice de gravedad propuesto por GesEPOC.

\section{Conflicto de intereses}

Los autores declaran no tener ningún conflicto de intereses.

\section{Bibliografía}

1. Lozano R, Naghavi M, Foreman K, Lim S, Shibuya K, Aboyans V, et al. Global and regional mortality from 235 causes of death for 20 age groups in 1990 and
2010: a systematic analysis for the Global Burden of Disease Study 2010. Lancet. 2012;380:2095-128.

2. Miravitlles M, Soriano J, García-Río F, Muñoz L, Duran-Tauleria E, Sanchez G, et al. Prevalence of COPD in Spain: impact of undiagnosed COPD on quality of life and daily life activities. Thorax. 2009;64:863-8.

3. Miravitlles M, Soler-Cataluña JJ, Calle M, Molina J, Almagro P, Quintano JA, et al. Spanish COPD Guidelines (GesEPOC): pharmacological treatment of stable COPD Spanish Society of Pulmonology and Thoracic Surgery. Arch Bronconeumol. 2012;48:247-57.

4. Miravitlles M, Soler-Cataluña JJ, Calle M, Molina J, Almagro P, Quintano JA, et al. Spanish Guidelines for Management of Chronic Obstructive Pulmonary Disease (GesEPOC) 2017. Pharmacological treatment of stable phase. Arch Bronconeumol. 2017;53:324-35.

5. From the Global Strategy for the Diagnosis, Management and Prevention of COPD, Global Initiative for Chronic Obstructive Lung Disease (GOLD) 2017. Disponible en: http://goldcopd.org.

6. Soler-Cataluña JJ, Martinez-Garcia MA, Sanchez LS, Tordera MP, Sanchez PR. Severe exacerbations and BODE index: two independent risk factors for death in male COPD patients. Respir Med. 2009;103:692-9.

7. Celli BR, Cote CG, Marin JM, Casanova C, Montes de Oca M, Mendez RA, et al. The body-mass index, airflow obstruction, dyspnea, and exercise capacity index in chronic obstructive pulmonary disease. N Engl J Med. 2004; 350:1005

8. Celli B, MacNee W, Agusti A, Anzueto A, Berg B, Buist A, et al. Standards for the diagnosis and treatment of patients with COPD: a summary of the ATS/ERS position paper. Eur Respir J. 2004;23:932-46.

9. Miller M, Hankinson J, Brusasco V, Burgos F, Casaburi R, Coates A, et al. Standardisation of spirometry. Eur Respir J. 2005;26:319-38.

10. Burge S, Wedzicha JA. COPD exacerbations: definitions and classifications. Eur Respir J. 2003;21 Suppl. 41:46S-53S.

11. Bestall J, Paul E, Garrod R, Garnham R, Jones P, Wedzicha J. Usefulness of the Medical Research Council (MRC) dyspnoea scale as a measure of disability in patients with chronic obstructive pulmonary disease. Thorax. 1999;54: 581-6.

12. Charlson M, Szatrowski T, Peterson J, Gold J. Validation of a combined comorbidity index. J Clin Epidemiol. 1994;47:1245-51.

13. ATS Committee on Proficiency Standards for Clinical Pulmonary Function Laboratories. ATS statement: guidelines for the six-minute walk test. Am J Respir Crit Care Med. 2002;166:111-7.

14. Bilder C, Zhang B, Schaarschmidt F, Tebbs J. binGroup: a package for group testing. R J. 2010;2:56-60.

15. Golpe R, Suárez-Valor M, Castro-Añón O, Perez-de-Llano LA. Risk stratification in chronic obstructive pulmonary disease. Can the Spanish guidelines be improved? Arch Bronconeumol. 2018;54:533-5.

16. Sin DD, Wu L, Man SF. The relationship between reduced lung function and cardiovascular mortality: a population-based study and a systematic review of the literature. Chest. 2005;127:1952-9.

17. Ma Z, Liu Y, Xu Y, Huang Y, Xu M, Zhu X, et al. Impaired lung function is associated with increased carotid intima-media thickness in middle-aged and elderly Chinese. PLoS One. 2013;8:e53153.

18. Sin D, Man S. Chronic obstructive pulmonary disease as a risk factor for cardiovascular morbidity and mortality. Proc Am Thorac Soc. 2005;2:8-11.

19. Cabrera C, Casanova C, Martín Y, Mirabal V, Sánchez MC, Álvarez F et al. Agreement between a simple dyspnea-guided treatment algorithm for stable COPD and the GOLD guidelines: a pilot study. Int J Chron Obstruct Pulmon Dis. 2016;11:1217-22

20. Soler-Cataluña J, Martínez-García M, Román Sánchez P, Salcedo E, Navarro M, Ochando R. Severe acute exacerbations and mortality in patients with chronic obstructive pulmonary disease. Thorax. 2005;60:925-31.

21. Wedzicha J, Seemungal T. COPD exacerbations: defining their cause and prevention. Lancet. 2007;370:786-96.

22. Miravitlles M, Ferrer M, Pont A, Zalacain R, Alvarez-Sala JL, Masa F, et al. Effect of exacerbations on quality of life in patients with chronic obstructive pulmonary disease: a 2 year follow up study. Thorax. 2004;59: 387-95.

23. Cabrera López C, Casanova Macario C, Marín Trigo JM, de-Torres JP, Sicilia Torres R, González JM, et al. Comparison of the 2017 and 2015 Global Initiative for Chronic Obstructive Lung Disease. Reports impact on grouping and outcomes. Am J Respir Crit Care Med. 2018;197:463-9.

24. Lange P, Marott J, Vestbo J, Olsen K, Ingebrigtsen T, Dahl M, et al. Prediction of the clinical course of chronic obstructive pulmonary disease, using the new GOLD classification: a study of the general population. Am J Respir Crit Care Med. 2012;186:975-81. 